\title{
PERFIL DE PESSOAS ESTOMIZADAS DE UMA REGIÃO DE SAÚDE MINEIRA
}

Juliano Teixeira Moraes ${ }^{1}$, Raquel Silva Assunção², Francisco dos Santos de Sá ${ }^{3}$, Eduardo Ribeiro Lessa ${ }^{4}$, Leoni dos Santos Corrêa ${ }^{4}$

Objetivo: descrever o perfil epidemiológico das pessoas estomizadas de uma região de saúde do estado de Minas Gerais. Metodologia: estudo descritivo, transversal, realizado de agosto a novembro de 2014, no Serviço de Atenção à Saúde da Pessoa Ostomizada de nível II do município de Divinópolis. Os dados foram colhidos mediante análise dos prontuários utilizados para cadastro e acompanhamento dessas pessoas no serviço de saúde e submetidos à análise descritiva. Resultados: analisados 185 prontuários de pessoas estomizadas, identificou-se a predominância de homens, casados, idosos, com baixo grau de instrução e com renda inferior a dois salários mínimos; estomias do tipo colostomias decorrentes do câncer de colo e reto. Conclusão: esse trabalho possibilitou ampliar o conhecimento da população de estomizados, podendo auxiliar o profissional de saúde, em especial o enfermeiro, a planejar a assistência do serviço de referência.

Descritores: Estomias; Perfil de saúde; Serviços de saúde.

\section{OSTOMIZED PROFILE OF A HEALTH REGION OF MINAS GERAIS STATE, BRAZIL}

Objective: to describe the epidemiological profile of stomized people in a health region of Minas Gerais. Methodology: descriptive, crosssectional study conducted from August to November 2014, the Care Health Service to the Stomized People level II of the municipality of the Divinópolis city. Data were collected by analyzing the records used for registration and monitoring of these people in the health service and submitted to descriptive analysis. Results: the study included 185 records of stomized people identified the predominance of men, married, older, low level of education and income less than two minimum salary; of the ostomy kind of colostomy resultin from the colon and rectum cancer. Conclusion: this work made it possible to expand the knowledge about the ostomy population and may aid health professionals, especially nurses, to plan the assistance of the reference service.

Descriptors: Ostomy; Health profile; Health service;

\section{PERFIL DE LAS PERSONAS CON ESTOMİA EN UNA REGIÓN SANITARIA DEL MINAS GERAIS, BRAZIL}

Objetivo: describir el perfil epidemiológico de las personas estomizadas en una región sanitaria de Minas Gerais. Metodología: estudio descriptivo, transversal, realizado entre agosto y noviembre de 2014, el Servicio de Salud de Atención del Personas Estomizadas nivel II del municipio de la ciudad de Divinópolis. Los datos fueron recolectados mediante el análisis de los registros utilizados para el registro y seguimiento de estas personas en el servicio de salud y sometidos a análisis descriptivo. Resultados: el estudio incluyó 185 registros de personas identificadas estomizadas el predominio de los hombres, casados, más viejo, bajo nivel de educación e ingresos de menos de dos salarios mínimos; del colostomia resultin del cáncer de colon y el recto. Conclusión: este trabajo ha permitido ampliar el conocimiento sobre la población de ostomia, puedendo ayudar a los profesionales de la salud, sobre todo enfermeras, para planificar la asistencia del servicio de referencia.

Descriptores: Estomía; Perfil de salud; Servicios de salud; 


\section{INTRODUÇÃO}

A pessoa estomizada é aquela submetida a uma cirurgia que resulta na confecção de um estoma. O termo estoma ou estomia tem origem na palavra grega "stoma" e significa "abertura de origem cirúrgica de uma nova "boca", visando à exteriorização de qualquer víscera oca existente no corpo"(1). Os estomas mais comuns são os urinários e os intestinais. Os urinários são comumente denominados de derivações urinárias e são realizados em pacientes com doenças que envolvem a pelve renal, ureteres, bexiga e uretra, e tem por objetivo preservar a função renal ${ }^{(1,2)}$. Os estomas intestinais são indicados quando alguma parte do intestino apresenta disfunção, obstrução ou lesão. O estoma recebe o nome de acordo com a porção intestinal, podendo ser ileostomia, cecostomia ou colostomia(2,3).

Estomias intestinais podem ser realizadas por doenças benignas ou malignas, criadas em condições de cirurgia eletiva ou de emergência, construídas no intestino delgado ou colón, podendo ser consideradas temporária ou permanente, e ainda para fins curativos ou paliativos ${ }^{(4)}$. Esses estomas são causados por traumatismos, doenças congênitas, doenças inflamatórias (doença de Crohn, retocolite ulcerativa crônica, diverticulite), tumores e câncer do intestino (cólon) ${ }^{(5)}$.

As pessoas estomizadas, embora possuidoras de características comuns que as unem em um grupo especial, possuem necessidades e reações próprias implicitas na sua identidade e subjetividade. Assim, a resposta a essa problemática, causada pela abertura do estoma, guarda relação com as condições pessoais de cada um, bem como com as variações externas, tais como, a qualidade de suporte familiar, financeiro e assistencial recebidos em todas as fases de tratamento gerador de estoma(b).

Entretanto, poucos estudos têm demonstrado preocupação em descrever o perfil dessa clientela, uma vez que é difícil precisar um quadro epidemiológico sobre as estomias por serem sequelas ou consequências de doenças ou traumas e não uma doença. Considera-se ainda que os dados sobre a situação dos estomizados em nosso país, apresentados pelo Ministério da Saúde (MS), são incipientes ${ }^{(7)}$. Portanto, esse artigo teve como objetivo descrever o perfil epidemiológico das pessoas estomizadas de uma região de saúde do estado de Minas Gerais.

É importante conhecer esse perfil para que se possa subsidiar o planejamento e a gestão dos serviços de saúde, bem como ações de educação em saúde e capacitação dos profissionais da rede, com vistas à melhoria da assistência à saúde e consequentemente da qualidade de vida dos individuos.

\section{METODOLOGIA}

Trata-se de uma pesquisa transversal realizada no Serviço de Atenção à Saúde da Pessoa Ostomizada de nível II (SASPO II) do município de Divinópolis, por meio da análise de informações registradas em prontuários utilizadas para cadastro e acompanhamento dessas pessoas no serviço de saúde. A coleta de dados aconteceu no período de agosto a novembro de 2014.

O SASPO II é referência para a Região de Saúde de Divinópolis/Santo Antônio do Monte, Minas Gerais. A Região de Saúde de Divinópolis/Santo Antônio do Monte compreende uma extensão territorial de $6.601,7 \mathrm{Km}^{2}$, com uma população estimada em 433.163 habitantes no Centro-Oeste mineiro. Atende, portanto, as pessoas estomizadas encaminhadas por diversos municípios: Araújos, Arcos, Carmo do Cajuru, Cláudio, Divinópolis, Itapecerica, Japaraíba, Lagoa da Prata, Pedra do Indaiá, Perdigão, Santo Antônio do Monte, São Gonçalo do Pará e São Sebastião do Oeste ${ }^{(8)}$.

É um serviço que possui uma equipe multidisciplinar composta por médico, enfermeiro, assistente social, psicólogo e nutricionista. Presta assistência especializada e de natureza interdisciplinar às pessoas com estoma, objetivando sua reabilitação, incluindo a orientação para o autocuidado, prevenção, tratamento de complicações nas estomias, capacitação e fornecimento de equipamentos coletores e adjuvantes de proteção e segurança( ${ }^{(9)}$.

Assim, foram analisados 175 prontuários das pessoas estomizadas. Utilizou-se, como critério de inclusão, os prontuários de usuário ativo no SASPO II de Divinópolis, desde a sua implantação em maio de 2011 . Foram considerados prontuários inativos aqueles vinculados a usuários que se desligaram do serviço por motivo de óbito, reversão cirúrgica do estoma ou abandono do tratamento. Foram colhidas as seguintes variáveis de estudo no prontuário: município, idade, sexo, estado civil, grau de instrução, raça/cor, renda familiar, diagnóstico, tipo de estoma, permanência do estoma, localização, complicações, tipo de abdome, eliminação de efluentes e tipo de dispositivo indicado.

Todos os prontuários possuem ficha cadastral que compreende a avaliação de enfermagem e médica no momento de adesão ao sistema. Foi feita uma análise estatística descritiva de todas as informações apresentadas por meio de tabelas de frequências.

Os dados foram coletados após aprovação da Secretaria Municipal de Saúde de Divinópolis-MG e do Comitê de Ética da Universidade Federal de São João Del-Rei/ Campus Centro Oeste por meio do parecer n. 714.992 (CAAE: 32873614.0.0000.5545). O estudo respeitou os preceitos éticos determinados pela Resolução CONEP n 466/2012, garantindo o anonimato dos indivíduos participantes. Por se tratar de dados secundários, não foi aplicado o Termo de Consentimento Livre e Esclarecido, já que não houve contato ou outra forma de comunicação com as pessoas envolvidas na pesquisa.

\section{RESULTADOS}

A região de saúde de Divinópolis/Santo Antônio do Monte possui um número de 175 pessoas estomizadas cadastradas, com maior prevalência de cadastros oriundos do município de Divinópolis $(55,4 \%)$ (Tabela 1$)$. 
Tabela 1 - Distribuição de pessoas estomizadas cadastradas no serviço a cada 10.000 habitantes e porcentagem em relação ao total, por município da Região de Saúde de Divinópolis/Santo Antonio do Monte - MG, 2014

\begin{tabular}{lllll} 
Municipio & População & $\mathbf{N}^{\circ}$ & $\%$ & $\begin{array}{l}\text { Prevalência } \\
/ 1000 \text { habitantes }\end{array}$ \\
Araújos & 8.645 & 0 & 0 & 0,0 \\
Arcos & 38.946 & 15 & 8,6 & 3,85 \\
Carmo do Cajurú & 21.519 & 9 & 5,1 & 4,18 \\
Cláudio & 27.579 & 11 & 6,3 & 4,00 \\
Divinópolis & 228.643 & 97 & 55,4 & 4,24 \\
Itapecerica & 22.082 & 6 & 3,4 & 2,71 \\
Japaraíba & 4.206 & 3 & 1,7 & 7,13 \\
Lagoa da Prata & 49.654 & 10 & 5,7 & 2,01 \\
Pedra do Indaiá & 4.013 & 3 & 1,7 & 7,48 \\
Perdigão & 10.185 & 2 & 1,1 & 1,96 \\
Santo Antônio & 27.556 & 14 & 8,0 & 5,08 \\
do Monte & & & & \\
São Gonçalo do Pará & 11.475 & 5 & 2,9 & 4,36 \\
São Sebastião do Oeste & 6.344 & 0 & 0 & 0 \\
\hline & & 175 & 100,0 & \\
\hline
\end{tabular}

Fonte: SASPO nivel II de Divinópolis / IBGE

Ainda, levando-se em consideração a população geral de cada município, a prevalência dos casos mostra Pedra do Indaiá, Japaraiba e Santo Antônio do Monte, como os municípios com maior prevalência de pacientes estomizados acompanhados no serviço de referência.

O perfil sócio-demográfico descreve uma população com maior representatividade no sexo masculino (55,4\%). Os dados mostram que a situação conjugal mais frequente é de pessoas casadas (42,9\%). Com relação à idade, houve maior ocorrência de pessoas acima de 60 anos (63\%). O grau de instrução, levando-se em consideração o tempo de estudo, demonstrou a predominância de pessoas semianalfabetas, ou seja, aqueles que sabem ler e escrever, mas não concluíram o ensino fundamental (29,7\%) e com ensino fundamental (24\%). Em relação à raça, após utilizar o critério preconizado pelo Instituto Brasileiro de Geografia e Estatística (IBGE), houve predomínio de pacientes declarados brancos (65,1\%). Por fim, a renda familiar, expressa em salários mínimos, descreve a população com renda de um a dois salários mínimos (49,1\%) (Tabela 2).

Tabela 2 - Características socioeconômicas e demográficas dos usuários acompanhados pelo Serviço de Atenção à Saúde das Pessoas Estomizadas. Divinópolis, 2014. (n=175)

\begin{tabular}{lll} 
Variáveis & $\mathbf{n}$ & $\%$ \\
\hline Sexo & & \\
Masculino & 97 & 55,4 \\
Feminino & 78 & 44,6
\end{tabular}

\begin{tabular}{|c|c|c|}
\hline \multicolumn{3}{|l|}{ Estado civil } \\
\hline Casado(a) & 75 & 42,9 \\
\hline Solteiro(a) & 28 & 16,0 \\
\hline Viúvo(a) & 27 & 15,4 \\
\hline Divorciado & 08 & 4,6 \\
\hline Amasiado(a) & $\mathrm{Ol}$ & 0,6 \\
\hline Não informado & 36 & 20,5 \\
\hline \multicolumn{3}{|l|}{ Idade } \\
\hline 80 e mais & 28 & 16,2 \\
\hline 60 a 79 anos & 81 & 46,8 \\
\hline 20 a 59 anos & 54 & 31,2 \\
\hline$<20$ anos & 10 & 5,8 \\
\hline Não informado & 02 & 0,1 \\
\hline \multicolumn{3}{|l|}{ Grau de Instrução } \\
\hline Analfabeto(a) & 17 & 13,6 \\
\hline Semianalfabeto(a) & 52 & 41,6 \\
\hline Nivel fundamental & 42 & 33,6 \\
\hline Nivel médio & 10 & 8,0 \\
\hline Nivel superior & 04 & 3,2 \\
\hline Não informado & 50 & 28,5 \\
\hline \multicolumn{3}{|l|}{ Renda familiar } \\
\hline < l salário mínimo & 13 & 12,04 \\
\hline la $<2$ salários mínimos & 86 & 79,63 \\
\hline $2 \mathrm{a}<3$ salários mínimos & 06 & 5,55 \\
\hline 3 a $<4$ salários mínimos & 03 & 2,78 \\
\hline Não informado & 67 & 89,3 \\
\hline
\end{tabular}

Fonte: SASPO nivel II de Divinópolis / IBGE

O tipo de estomia mais prevalente foi a colostomia (73,7\%). A principal causa para realização do estoma observada foi a decorrente de Neoplasia Colorretal (46,9\%). Nãoforam observadas complicações registradas em $58 \%$ das pessoas vinculadas ao serviço. As complicações registradas apontaram para prolapso (11,36\%), retração (10,79\%) e dermatite (9,1\%) (Tabela 3).

Tabela 3 - Estomias, diagnósticos e tipos de complicações dos usuários acompanhados pelo Serviço de Atenção à Saúde das Pessoas Estomizadas. Divinópolis, 2014. (n=175)

\begin{tabular}{lll} 
Variáveis & $\mathbf{n}$ & $\%$ \\
\hline Tipo de Estomia & & \\
\hline Colostomia Esquerda & 101 & 58,4 \\
Colostomia Direita & 28 & 16,1 \\
Ileostomia & 24 & 13,9 \\
Urostomia & 19 & 11,0 \\
Fístula & 01 & 0,6 \\
Não informado & 02 & 1,1 \\
Diagnóstico & & \\
Neoplasia Colorretal & 82 & 48,8 \\
Outros & 43 & 25,6 \\
Doença Diverticular & 12 & 7,1
\end{tabular}




$\begin{array}{lll}\text { Megacolon/ Colón Chagásico } & 11 & 6,6 \\ \text { Megacolon congênito (Hirschisprung) } & 05 & 3,0 \\ \text { Doença inflamatória intestinal } & 04 & 2,4 \\ \text { Volvo } & 04 & 2,4 \\ \text { Trauma } & 04 & 2,4 \\ \text { Complicações de apendicite } & 02 & 1,2 \\ \text { Colite isquêmica } & 01 & 0,6 \\ \text { Não informado } & 07 & 4,8 \\ \text { Complicação } & & \\ \text { Sem complicação } & 102 & 58,29 \\ \text { Prolapso } & 20 & 11,43 \\ \text { Retração } & 19 & 10,86 \\ \text { Dermatite } & 16 & 9,14 \\ \text { Hérnia Paracolostômica } & 09 & 5,14 \\ \text { Edema } & 03 & 1,71 \\ \text { Descolamento Mucocutâneo } & 03 & 1,71 \\ \text { Sangramento } & 02 & 1,14 \\ \text { Abscesso } & 01 & 0,57\end{array}$

Fonte: SASPO nivel II de Divinópolis / IBGE

Os dados referentes ao tempo de permanência com o estoma (temporária ou permanente) não foram analisados, uma vez que essa informação foi auto relatada pelo estomizado ao serviço, não sendo constatada a indicação médica.

\section{DISCUSSÃO}

Embora o número de estomizados tenha aumentado nos últimos anos, percebe-se que nessa Região de Saúde, a prevalência de estomizados, identificada nos prontuários, está abaixo do estabelecido pela IOA e ABRASO (1/1.000 habitantes) (6). Os baixos números registrados podem ser explicados pelo fato de que se trata de um serviço com menos de cinco anos de existência na região, muitos usuários podem não conhecer o programa ou não estar vinculados aos serviços públicos de saúde(10).

Nessa população, ao se comparar o número de estomizados de cada município com a população geral, observou-se que municipios de pequeno porte tiveram uma prevalência proporcionalmente maior em relação aos municípios maiores. Novos estudos devem ser realizados para se compreender melhor essa população, afim de se conhecer e analisar as condições que fazem com que esse número de casos seja maior em relação aos outros municipios.

O avançar da idade é um dos fatores que mais se destaca no processo de oncogênese em decorrência da maior exposição a fatores de risco ao longo dos anos; consequentemente, o número de estomias cresce nessa população(11). A população de idosos estomizados revela implicações para o cuidado. Sabe-se que eles podem sofrer mais impactos psicológicos como resultado de estar estomizado. Os profissionais de saúde devem entender as mudanças relacionadas ao envelhecimento e como elas podem afetar o autocuidado das pessoas estomizadas. Deve ser considerado, ainda, que os homens parecem ter mais dificuldade com ajustamento emocional após a cirurgia, quando comparados com as mulheres mais idosas com ostomias ${ }^{(12)}$.

Além disso, deve-se considerar que 0 homem busca menos os serviços de saúde para prevenção de agravos. Dados do Ministério da Saúde (MS) revelam que os homens apenas procuram o Sistema Único de Saúde (SUS) por meio da atenção especializada, tendo, como consequência, o agravo da morbidade pelo retardamento na atenção e maior custo para o SUS. O modelo de uma masculinidade ainda idealizada consiste numa ideia de invulnerabilidade e, portanto, de comportamento de risco. Associado a isso, encontram-se suas dificuldades de verbalizar as próprias necessidades de saúde, pois falar de seus problemas de saúde pode significar uma possivel demonstração de fraqueza, de feminilização perante os outros ${ }^{(13)}$.

Embora a população tenha se destacado entre idosos, destacou-se, também, o número de pessoas casadas. Sendo casadas, há de se considerar a sexualidade vivenciada por esse grupo. A presença de enfermidade, tanto no parceiro como no próprio idoso, tem sido o principal fator envolvido para a ausência ou diminuição da sexualidade, principalmente quando a doença ocorre no homem, pois comumente afeta a potência masculina ${ }^{(14)}$.

A convivência com a bolsa coletora leva ao aparecimento de sentimentos conflituosos, preocupações e dificuldade para lidar com essa nova situação(15). A presença de um parceiro para a pessoa com estomia contribui para manter uma atitude de esperança realista, além de poder partilhar preocupações e receios, afim de ajudar-se mutuamente ${ }^{(16)}$

O grau de instrução encontrado nesse estudo deve ser bastante considerado, devido aos necessários esclarecimentos sobre a doença e o tratamento, e assim, garantir a adesão dos pacientes ao autocuidado. A baixa escolaridade pode refletir-se na forma de assimilar as orientações a serem transmitidas acerca dos cuidados com o estoma ${ }^{(17)}$.

No Brasil, o câncer colorretal está entre as quatro localizações mais frequentes de neoplasias, em ambos os sexos. Abrange tumores que acometem um segmento do intestino grosso (o cólon) e o reto. Para 2016, a estimativa de novos casos é de $34.280^{(18)}$. O tratamento do câncer colorretal consiste em procedimento cirúrgico, quimioterapia e radioterapia, sendo as duas últimas terapias associadas à cirurgia. A ressecção cirúrgica do local afetado e a realização de uma colostomia constituem-se na mais efetiva terapia para o câncer colorretal ${ }^{(19)}$

O número predominante de pessoas colostomizadas em relação às ileostomizadas faz com que, ao planejar assistência, algumas considerações sejam observadas: menor número de lesões peri-estomais, possibilidade de se realizar a irrigação, consistência das fezes, maior tempo entre alimentação e evacuação, condição de absorção de alimentos e medicamentos, dentre outros ${ }^{(3)}$.

Complicações do tipo prolapso e retração dizem respeito principalmente ao posicionamento da estomia quando do procedimento cirúrgico. O aparecimento dessas complicações pode ser consequência de consultas pré-operatórias sem 
demarcação de estoma. A má localização dificulta o autocuidado, a visualização da pele e a troca de equipamentos ${ }^{(20)}$.

Considera-se como limitação desse estudo a deficiência identificada nos registros de informações.

\section{CONCLUSÃO}

Este trabalho possibilitou ampliar o conhecimento da população de estomizados da Região de Saúde de Divinópolis/ Santo Antônio do Monte, Minas Gerais. Essa população mostrouse como predominantemente de homens, casados, idosos, com baixo grau de instrução e com renda inferior a dois salários mínimos.

Houve predomínio de estomias do tipo colostomias decorrentes do câncer de colo e reto. Dentre as complicações, o estudo chama a atenção para o prolapso, retração e dermatite periestomia.

Estudos dessa natureza poderão auxiliar o profissional de saúde, em especial o enfermeiro, a planejar a assistência do serviço de referência. Dessa forma, atividades educativas e atendimentos clínicos poderão ser melhor compreendidos pelos estomizados, uma vez que se compreende melhor o seu contexto.

\section{AGRADECIMENTOS}

Ao Programa de Educação pelo Trabalho para a Saúde Redes de Atenção à Saúde (PET-Redes) da Secretaria de Gestão do Trabalho e da Educação na Saúde do Ministério da Saúde.

\section{REFERÊNCIAS}

1. Fernandes RM, Borges EL, Donoso MTV. Perfil da clientela estomizada residente no municipio de Ponte Nova Minas Gerais. Rev bras coloproctol. 2011 [citado em 2016 abr 22]; 30(4): 385-392. Disponivel em: http://www.scielo.br/scielo.php?script=sci_arttextEpid=SO1O1$988020100004000018 \operatorname{lng}=e n$

2. Recalla S1, English K, Nazarali R, Mayo S, Miller D, Gray M. Ostomy care and management: a systematic review. J Wound Ostomy Continence Nurs. 2013 Sep-Oct; 40(5):489-500.

3. Registered Nurses Association Of Ontario (RNAO). Ostomy care and management. Toronto: Registered Nurses'Association of Ontario; 2009. Disponivel em: http://rnao.ca/sites/rnao-ca/files/Ostomy_Care_Management.pdf

4. Hendren S, Hammond, K, Glasgow SC, Perry WB, Buie WD et al. Clinical Practice Guidelines for Ostomy Surgery. Dis Colon Rectum 2015 [citado em 2016 abr 22]; 58: 375-387. Disponivel em: https://www.fascrs.org/sites/ default/files/downloads/publication/clinical_practice_guidelines_for_ ostomy_surgery.pdf

5. Kleinubing-Júnior H, Pinho Mauro SL, Ferreira LC, Bachtold GA, Merki A. Perfil dos pacientes ambulatoriais com doenças inflamatórias intestinais. ABCD, arq. bras. cir. dig. 2011 [citado em 2016 Abr 22]; 24(3): 200-203. Disponivel em: http://www.scielo.br/scielo.php?script=sci_ arttextEpid=S0102-67202011000300004\&lng=en.

6. Santos VLCG, Cesaretti IUR. Assistência em estomaterapia - cuidando de pessoas com estomia: 2ed. Rio de Janeiro: Atheneu: 2015.

7. Santos VLCG. Aspectos Epidemiológicos dos Estomas. Estima. 2007 [citado em 2016 jun 24]; 5(1). Disponivel em: http://www.revistaestima.com. br/index.php/estima/article/view/207.

8. Secretaria de Estado de Saúde. Assessoria de Gestão Regional (Minas Gerais). O PDR - Plano Diretor de Regionalização da Saúde de Minas Gerais. Belo Horizonte: Secretaria de Estado de Saúde, 2010.

9. Ministério da Saúde. Secretaria de Assistência a Saúde (Brasil). Portaria $\mathrm{n} .400$ de 16 de novembro de 2009. Institui a atenção à saúde da pessoa estomizada. Diário Oficial da Uniáo 18 nov. 2009; 220(1): 41-42.

10. Sampaio FAA, Aquino PS, Araújo TL, Galvão MTG. Nursing care to an ostomy patient: application of the Orem's theory. Acta paul. enferm. 2008 [citado em 2016 abr 22]; 21(1): 94-100. Disponivel em: http://www.scielo.br/ scielo.php?script=sci_arttext\&pid=S0103-21002008000100015\&lng=en.

11. Da Paz RC, Fortes RC, Toscano BAF. Processo de envelhecimento e câncer: métodos subjetivos de avaliação do estado nutricional em idosos oncológicos. Com. Ciências Saúde. 2011 [citado em 2016 abr 22]: 22(2):143-
156. Disponivel em: http://www.escs.edu.br/pesquisa/revista/2011Vol\%20 22_2_6_Processo.pdf.

12. Skeps R, McMullen CK, Wendel CS, Bulkley J, Grant M, Mohler J et al. Changes in Body Mass Index and Stoma Related Problems in the Elderly. Journal of geriatric oncology. 2013 [citado em 2016 abr 22] 4(1):84-89. Disponivel em: http://www.ncbi.nlm.nih.gov/pmc/articles/ PMC3564631/?tool=pubmed.

13. Da Silva PLN, Maciel MM, Carfesan CS, Santos S, Souza JR. Politica de atenção à saúde do homem no Brasil e os desafios da sua implantação: uma revisão integrativa. Enferm. glob. 2013 [citado em 2016 abr 22] 12(32): 381-413. Disponivel em: http://scielo.isciii.es/scielo.php?script=sci_ arttext\&pid=S1695-61412013000400024\&lng=es.

14. Alencar DL, Marques APO, Leal MCC. Vieira JCM. Fatores que interferem nasexualidade deidosos: uma revisão integrativa. Ciênc. saúde coletiva. 2014 [citado em 2016 Abr 22]; 19(8): 3533-3542. Disponivel em: http://www.scielo. br/scielo.php?script=sci_arttextEpid=S1413-81232014000803533\&lng=en.

15. Cardoso DBR, Almeida CE, Santana ME, Carvalho DS, Sonobe HM. Sawada NO. Sexualidade de pessoas com estomias intestinais. Rev Rene. 2015 [citado em 2016 Abr 22]: 16(4): 576-85. Disponivel em: http://www. revistarene.ufc.br/revista/index.php/revista/article/viewFile/2051/pdf.

16. Sousa CF, Brito DC, Branco MZPC. Depois da colostomia... vivências das pessoas portadoras. Enfermagem em Foco 2012 [citado em 2016 Abr 22]; 3(1):12-15. Disponivel em: http://revista.cofen.gov.br/index.php/ enfermagem/article/viewFile/213/134.

17. Menezes LCG, Guedes MVC, Oliveira RM, Oliveira SKP, Meneses LST, Castro ME. Prática de autocuidado de estomizados: contribuições da teoria de orem Rev Rene. 2013 [citado em 2016 Abr 22]: 14(2): 301-10. Disponivel em: http://www.revistarene.ufc.br/revista/index.php/revista/article/ view/235

18. Instituto Nacional de Câncer José Alencar Gomes da Silva (INCA). Coordenação de Prevenção e Vigilância. Estimativa 2016: incidência de câncer no Brasil: INCA; 2015.

19.Guimarães RM, Muzi CD, Boccolini CS, Boccolini PMM, Boeira SF. Tendência da mortalidade por câncer de cólon e reto no Brasil segundo sexo, 1980-2009 Cad. Saúde Colet. 2012 [citado em 2016 Abr 22]; 20(1): 121-7. Disponivel em: http://www.cadernos.iesc.ufrj.br/cadernos/images/ csc/2012_1/artigos/CSC_v2Onl_121-127.pdf.

20. Miranda SM Luz MHBA Sonobe HM Andrade EMLR Moura ECC. Caracterização Sociodemográfi ca e Clinica de Pessoas com Estomia em Teresina. Estima. 2016 [citado em 2016 Abr 22]; 14(1): 29-35. Disponivel em: http://www.revistaestima.com.br/index.php/estima/article/view/117/pdf. 